\title{
Enseñanza de la lectura a través de phonics en el aula de Lengua Extranjera en Educación Primaria
}

\author{
Amparo LÁZARo IBARROLA \\ Universidad Pública de Navarra \\ Departamento de Filología y Didáctica de la Lengua y la Literatura
}

Recibido: 5-2-07 / Aceptado: 6-3-07

ISSN: $1697-7467$

\begin{abstract}
Over the last decade, Spanish EFL classrooms have changed dramatically: the onset age has gone down and the number of English sessions per week has increased. However, these changes have not always been accompanied by new teaching practices. A strong deficiency is the entire lack of method to assist Spanish children in EFL reading, which makes the process difficult and worsens the pronunciation due to L1 grapho-phonemic transfer. Within this context, we propose the use of phonics, phonological awareness methods designed to teach English natives to read, in EFL classrooms.
\end{abstract}

Key words: reading, EFL, phonics, phonology.

\begin{abstract}
En la última década el aula de inglés ha cambiado en España principalmente en dos aspectos: el descenso en la edad de comienzo y el incremento en el número de horas. Sin embargo, la práctica docente no siempre se ha adaptado a estos cambios. Una carencia es la falta de método para ayudar al alumno a enfrentarse al texto escrito, lo cual dificulta el proceso lector y perjudica la pronunciación, ya que el alumno transfiere las normas grafo-fonémicas del castellano. Por ello, proponemos la utilización de los métodos de concienciación fonológica denominados phonics para enseñar a nuestros alumnos a leer en inglés.
\end{abstract}

Palabras clave: lectura, inglés lengua extranjera, fonología.

\section{INTRODUCCIÓN}

En este trabajo presentamos una propuesta metodológica innovadora: la utilización de los materiales didácticos denominados phonics $^{l}$ para facilitar el aprendizaje de la lectura en inglés como lengua extranjera a los alumnos españoles de Educación Primaria. Los phonics son los métodos de lecto-escritura basados en la concienciación fonológica que se utilizan para enseñar a leer a los niños angloparlantes. Sin embargo, a pesar de la gran cantidad de trabajos centrados en el estudio del proceso lector y de la conciencia fonológica en L1, su papel en el aula de lengua extranjera no se ha investigado. Por ello, esta propuesta

${ }^{1}$ Optamos por mantener el término inglés. 
metodológica supondrá una aportación innovadora no solo para la práctica docente en sí misma, sino también para el desarrollo científico de áreas como la Didáctica de la Lengua Extranjera, la Lingüística Aplicada, la investigación sobre el proceso lector y la propia Fonética.

En el aula de inglés, la utilización de estos métodos con los alumnos de Educación Primaria cumpliría un doble objetivo: Por una parte, serviría para evitar la transferencia de las reglas grafo-fonémicas del castellano y así proteger y mejorar la pronunciación del inglés, idioma que, hasta el momento de enfrentarse al texto escrito, los niños han adquirido de manera exclusivamente oral. Por otra parte, los phonics servirían para facilitar el aprendizaje de la lectura en inglés al ayudar al alumno a asociar el texto escrito con el texto oral, favoreciendo así la comprensión lectora en este idioma. Además, desde un punto de vista didáctico más general, podríamos añadir que, al convertir el proceso lector en un proceso más asequible y menos dificultoso también cumplirían con el objetivo de motivar al alumno y crear (o reforzar) en él actitudes positivas tanto hacia el inglés como hacia la propia lectura. Si tomamos el punto de vista de los profesores, la utilización de los phonics viene a proporcionarles una nueva herramienta asequible, fácil de utilizar y de gran eficacia para sacar el mayor provecho posible del aula de inglés. Por supuesto, aunque nuestra propuesta se ubica en el aula de Educación Primaria en España, el interés de la misma alcanza a otros muchos países que también se enfrentan a la enseñanza del inglés en etapas tempranas. De hecho, el que no se haya investigado el proceso lector en la lengua extranjera se debe, en parte, al hecho de que, hasta hace pocos años, no se enseñaran las lenguas extranjeras de manera extensiva en edades tempranas.

Más allá del aula, como hemos dicho, esta propuesta metodológica supone la apertura de nuevas vías de investigación de gran interés en varios ámbitos, pero sobretodo, esperamos que nuestra propuesta abra la puerta a futuros estudios empíricos que sirvan para poner a prueba su validez.

\section{Contextualización}

\subsection{El inglés en contexto escolar}

Respondiendo a las necesidades de la sociedad, el aprendizaje de idiomas en general y del inglés en particular ha ido cobrando cada vez mayor entidad tanto en nuestro país como a nivel europeo. Así, la Comunidad Europea se ha implicado de lleno en este tema, como lo muestran iniciativas tales como el Año Europeo de las Lenguas (2001), el Plan de Acción para el Aprendizaje de las Lenguas y la Diversidad Lingüística (2004-2006) y, por supuesto, el Marco Común Europeo de Referencia para las Lenguas: aprendizaje, enseñanza, evaluación (2001), documento elaborado por el Consejo Europeo para superar las barreras de los distintos sistemas educativos y sentar unas bases comunes para la elaboración de programas de lenguas en toda Europa.

Del mismo modo, las autoridades educativas españolas no han permanecido ajenas a esta realidad y, a pesar de los sucesivos cambios en las Leyes Orgánicas de Educación (LOGSE 
(1990); LOCE (2002) y LOE (2006) $)^{2,3}$, la adquisición de competencia comunicativa en un idioma extranjero figura en todas ellas como un objetivo básico tanto en Educación Primaria como en Educación Secundaria. Por ejemplo, la LOE incluye como objetivo en Educación Primaria "adquirir en, al menos una lengua extranjera, la competencia comunicativa básica que les permita (a los alumnos) expresar y comprender mensajes sencillos y desenvolverse en situaciones cotidianas". (LOE, capítulo III, artículo 17 apartado (f)). Esta misma Ley incluye el siguiente objetivo para la Educación Secundaria: "Comprender y expresarse en una o más lenguas extranjeras de manera apropiada” (LOE, capítulo III, articulo 23, apartado (i)). Como novedad frente a la LOGSE, tanto en la LOCE como en la actual LOE, el idioma extranjero aparece mencionado por primera vez en la etapa de Educación Infantil del siguiente modo: "Las Administraciones educativas promoverán la incorporación de una lengua extranjera en los aprendizajes de la Educación Infantil, especialmente en el último año”. (LOCE, capítulo I, artículo 12, apartado 3).

Así pues, el área de Lengua Extranjera (inglés) ha ido cobrando cada vez mayor importancia en el currículo escolar, especialmente en la etapa de Educación Primaria, donde, a pesar de que se encuentran diferencias según el centro y la comunidad autónoma a que nos refiramos, en general, se han seguido las siguientes directrices:

- Se ha ido adelantando la edad de comienzo de aprendizaje de la lengua extranjera.

- Se ha aumentado el número de horas semanales.

- Ha cambiado la metodología.

- Se han renovado los materiales y recursos (audiovisuales, soportes informáticos).

- Se ha incrementado el uso del inglés en el aula.

- Se ha comenzado a utilizar el inglés como medio de instrucción.

- La asignatura de inglés ha pasado a impartirse exclusivamente por especialistas.

Para ilustrar estos cambios con datos concretos podemos tomar como ejemplo la Comunidad Foral de Navarra. En esta comunidad, la Orden Foral 230/1992 de 12 de junio establecía únicamente la enseñanza del inglés en los ciclos medio y superior de Educación Primaria, con una dedicación de tres horas semanales. Diez años después, en la siguiente Orden Foral (Orden Foral 366 de 5 de julio de 2002), se pasa a incluir el inglés en toda la Educación Infantil con cuatro sesiones semanales y en toda la Educación Primaria con cinco sesiones, de las cuales dos deben dedicarse a impartir materias en inglés.

En realidad, este incremento tan espectacular de la presencia del inglés en la escuela no venía sino a responder a lo que era ya una demanda social y de los propios centros escolares que, en muchos casos, habían ido incrementando paulatinamente la presencia del idioma adelantándose a la Ley. En este nuevo contexto, es evidente que la labor docente y formación académica de un maestro que impartía tres horas de inglés a la semana en un aula de segundo ciclo de Educación Primaria es insuficiente e inadecuada. Por ello, es necesaria una adaptación a esta nueva realidad tanto de los Maestros como de las Universidades y otros organismos encargados de su formación.

\footnotetext{
${ }^{2,3}$ Ley Orgánica de Ordenación General del Sistema Educativo (1/1990, de 3 de Octubre) (LOGSE). Ley Orgánica de Calidad de la Educación (10/2002 de 23 de diciembre) (LOCE).

Ley Orgánica de Educación (2/2006, de 3 de mayo) (LOE).
} 
Por un lado, se exige un enorme esfuerzo por parte del Maestro de inglés en activo que debe adaptarse a la nueva realidad del aula actualizando sus conocimientos, modificando la metodología, utilizando nuevos materiales etc. Por otro lado, esta adaptación afecta también a las Universidades (y otros organismos implicados en la formación de maestros), que deben ofrecer oportunidades de formación que sirvan para sacar el máximo rendimiento a la situación actual de la asignatura de inglés. Por ello, para lograr sacar el máximo provecho de las profundas reformas que ha sufrido el área de lengua extranjera en la escuela y de las enormes inversiones que se han realizado en recursos humanos y materiales, hoy, más que nunca, es fundamental que la labor de investigación universitaria y la labor docente se retroalimenten. Desafortunadamente, la magnitud y velocidad de los cambios ha provocado que, en muchos casos, ni el Maestro, ni las Universidades, hayan podido preparase adecuadamente para cumplir las expectativas creadas por las nuevas características que constituyen hoy en día el aula de inglés. En este sentido, en el preámbulo al Libro Blanco del Título de Grado de Magisterio ${ }^{4}$, Antonio Maldonado, coordinador de la Red de Magisterio, afirma lo siguiente: "La profunda reforma del Sistema Educativo que afectó a todos los niveles y dimensiones, sin duda hubiera sido mayor y más efectiva si se hubiera acompañado de una profunda reestructuración de las titulaciones de Profesorado, tanto de Ed. Infantil y Ed. Primaria, como de Ed. Secundaria”. (ANECA: 2004)

Esto no significa que no se hayan obtenido importantes logros que merece la pena destacar. Al contrario, el Profesor Antonio Maldonado en el Preámbulo del Libro Blanco continua: "Con todo, la creación de nuevas especialidades de Magisterio supuso una extraordinaria mejora en la formación en unos ámbitos (...) en los que nuestro sistema educativo no se encontraba en parámetros semejantes de los países de nuestro entorno, y además, había una clara demanda social de esta formación" (ANECA: 2004). Del mismo modo, es importante citar los numerosos trabajos de investigación para el aula que se vienen realizando en los últimos años (Madrid y McLaren, 2004 y Vez, Guillén y Alario, 2002 entre otros) cuyas aportaciones sobre didáctica del inglés son valiosísimas para la labor del docente. En la línea de estos trabajos, este artículo quiere ser una contribución a la mejora en la formación de maestros $\mathrm{y}$, consecuentemente, a la mejora del nivel de inglés en los colegios centrándose en un aspecto que, hasta ahora, se ha relegado a un segundo término, cuando no ignorado: la utilización didáctica de la fonética en Educación Primaria, más específicamente, su utilización en la enseñanza de la lectura a través de phonics.

\subsection{La formación del Maestro de Lengua Extranjera (inglés)}

Entre los puntos débiles recogidos en la Encuesta de Opinión a profesionales que figura en el anexo 6 del Libro Blanco de la Titulación de Maestro, el segundo punto se denomina "Adecuación Teoría/Realidad Escolar", etiqueta bajo la que" "se recogen fundamentalmente alusiones a la insuficiente presencia de la dimensión práctica de los contenidos de las

${ }^{4}$ Como en la introducción al Volumen I se dice, dicho libro muestra "el resultado del trabajo llevado a cabo por una red de universidades españolas, apoyadas por la ANECA, con el objetivo explícito de realizar estudios y supuestos prácticos útiles en el diseño de un título de grado adaptado al Espacio Europeo de Educación Superior (EEES)" 
asignaturas y la necesaria conexión de los aspectos de las distintas disciplinas con su proyección en criterios de actuación docente”. En nuestra opinión, precisamente una de las “áreas que no se explota en su dimensión práctica de cara a afrontar la docencia de la Lengua Extranjera en el aula es, sin duda, la Fonética. Por ello, con nuestro proyecto podemos contribuir a reforzar esta "dimensión práctica" de la que adolece, según los propios docentes, la formación de Maestros.

Tradicionalmente, se ha "asumido" que la pronunciación no requería de una formación específica, ni sistemática (enfoque explícito), sino que se iba adquiriendo de manera "natural" al ir aprendiendo el idioma (enfoque implícito) o, en ocasiones, su didáctica se reducía a la realización de actividades puntuales que en ningún caso formaban parte integrante del currículo. Sin embargo, cualquier docente estará de acuerdo en que la complejidad de la pronunciación de un idioma como el inglés, así como las carencias que presentan los alumnos (y en ocasiones los propios docentes) en comprensión y expresión oral, evidencian la necesidad de una formación más sólida en este aspecto. En nuestra opinión, parte de esta formación sólida la puede ofrecer la fonética con sus enormes (y muy inexploradas) posibilidades didácticas.

Desafortunadamente, los propios maestros de Lengua Extranjera desconocen muchas veces la existencia de estrategias y materiales de desarrollo de la conciencia fonológica puesto que este tipo de materiales ni aparecen en los libros de texto que habitualmente se utilizan en los colegios ni se incluyen en la formación del maestro que, generalmente, ni ha sido concienciado a cerca de la importancia y eficacia de trabajar la fonética en el aula, ni ha sido formado en la explotación de materiales didácticos para integrarla en su práctica docente. Probablemente, ni siquiera en su propio proceso de aprendizaje del inglés el Maestro ha realizado de manera sistemática actividades de índole fonética. En cualquier caso, queremos subrayar que la ausencia de formación en didáctica de la fonética supone, si no una carencia para el profesorado de lengua extranjera al menos, un no beneficiarse de un recurso que está disponible y que repercutiría positivamente en el aula de inglés.

Creemos, pues, que la explotación didáctica de la Fonética es hoy en día en nuestro país un área apenas explorada, que tiene mucho que aportar a la formación de maestros y, consecuentemente, a la enseñanza del inglés, ya que proporcionará al futuro Maestro de Lengua Extranjera las herramientas necesarias para que la enseñanza de la pronunciación se convierta en parte integrante del currículo en el aula de inglés. De entre las aportaciones posibles, escogemos una muy concreta: la aportación a la enseñanza de la lectura. Esta aportación viene, además, a contribuir a la enseñanza del inglés en un momento crucial: la etapa en la que el alumno de lengua extranjera pasa del texto oral al texto escrito. En este momento clave, nuestro proyecto pretende proporcionar a los maestros las herramientas necesarias para guiar al alumno en el aprendizaje de la lectura en inglés a través del desarrollo de la conciencia fonológica y adoptando y adaptando para ello las metodologías que utilizan los niños ingleses en su propio proceso de lecto-escritura: los phonics. 


\section{LA ENSEÑAnZA DE LA LECTURA}

\subsection{La conciencia fonológica en el aprendizaje de la lectura}

El paso del texto oral al texto escrito, que denominamos proceso de lecto-escritura ${ }^{5}$, es sin duda un proceso complejo en el que los alumnos requieren la guía del maestro. Este proceso ha sido muy trabajado en todas las lenguas, incluidas las implicadas en este trabajo: castellano e inglés (Alegría y Morais, 1979; Bryant y Bradley, 1998; Cassany, 1987, 1993; Clemente y Domínguez, 1999; Domínguez, 1996a,b; Graves, 1991; Pressley, 1999; Solé, 1992; Teberosky, 1991; Tolchinsky, 1993; entre otros).

Existen principalmente dos métodos de enseñanza lectora, el método fonológico y el método global, que cuentan con sus correspondientes defensores y detractores ${ }^{6}$. El método global se basa en un acceso directo al significado por vía visual, es decir, el alumno aprende a reconocer la palabra a partir de su imagen ortográfica sin necesidad de analizar el sonido al que corresponden cada una de sus grafías, mientras que el método fonológico accede primero a la identificación de las letras y sus sonidos y posteriormente, a partir de la construcción del sonido de la palabra a su significado. En nuestro trabajo optamos por el método fonológico ya que es el que más se adecua a las necesidades de nuestros alumnos por razones obvias: los niños ya saben leer en castellano y por tanto van a transferir las relaciones grafíasonido de la L1 y, además, nos interesa reforzar la pronunciación.

Dentro, pues, del método fonológico, a pesar de que existen diferentes opiniones a cerca de cómo se produce el proceso de aprendizaje de la lectura, casi nadie duda de la importancia de la fonología en el mismo7 (Byrne y Fielding-Barnsley, 1991; Carrillo y Sánchez-Meca, 1990; Cunningham, 1990; Jorm y Share, 1983; Read et al., 1986). De hecho, la propia naturaleza del sistema alfabético lo demuestra, ya que se trata de un sistema (orto)gráfico que se define como una representación fonológica de la lengua oral. En este orden de cosas se acuña el término conciencia fonológica (también denominada conocimiento metafonológico) que Tunmer (1989) define como la habilidad para reflexionar sobre los segmentos fonológicos del lenguaje oral.

Dos precisiones respecto al papel de esta conciencia fonológica en el proceso lector son de especial relevancia para nuestra propuesta: En primer lugar, el hecho de que la base fonológica del proceso lector se restringe al estadio inicial (Clemente, 2001; Johnston,1998; Scarborough et al. 1998). Según Clemente, al aprender a leer, recurrimos inicialmente a una vía fonológica que se define "como la reconversión oral que hacemos de cada letra con el correspondiente sonido de la lengua oral” (Clemente, 2001: 116). Sin embargo, en lectores avanzados la lectura pasa a basarse en su dimensión ortográfica, es decir, el lector identifica la palabra globalmente al ver sus grafías. En segundo lugar, destacar numerosos estudios que establecen una relación entre la conciencia fonológica y un buen nivel lector (Bradley y

${ }^{5}$ En este trabajo nos centramos exclusivamente en el proceso de lectura aunque, evidentemente, la escritura también saldrá beneficiada.

6 Ver Chall (1983) para una revisión de los diferentes métodos.

${ }^{7}$ Ver Scholes (1998) para una opinión diferente y la correspondiente respuesta que recibe de Johnston (1998). 
Bryant, 1983; Carrillo y Sánchez Meca, 1990; Cunningham, 1990; Domínguez 1996a; Liberman et al., 1974; Morais et al., 1984) y, en sentido inverso, la escasa conciencia fonológica con dificultades en la lectura (Juel, Griffith y Gough, 1986).

Una vez sentada la importancia de la conciencia fonológica en la etapa inicial y su relación con un buen nivel lector, surgen diferentes estudios que plantean cuestiones relevantes para nuestra propuesta. Así, como señalan Jiménez y Ortiz (1995), es necesario determinar la naturaleza de la relación entre conciencia fonológica y proceso lector, es decir, determinar si la conciencia fonológica es causa o consecuencia del aprendizaje de la lectura o bien, si se da una relación bidireccional entre ambas. A su vez, se plantea la cuestión de la necesidad o no de una instrucción explícita que desarrolle dicha conciencia en el niño. En este sentido existen estudios contradictorios. Por una parte, diversos autores (Morais et al., 1979; Read et al., 1986) han demostrado que la conciencia fonológica no se adquiere de manera espontánea, sino que es necesaria una intervención didáctica explícita. Por el contrario, Ehri y Wilce $(1980,1987)$ descubren que algunos niños son capaces de aprender a leer sin necesidad de reflexión fonológica. En cualquier caso, Griffith y Olson (1992), demostraron que, al margen del método utilizado para la enseñanza de la lectura, los niños que tenían mayor conciencia fonológica superaban al resto en habilidades lectoras. Es decir, aunque sea posible alcanzar la conciencia fonológica sin intervención didáctica explícita, parece innegable que ayudar al niño a adquirirla por medio de métodos didácticos facilitará el proceso de lectura, lo cual es sin duda deseable desde el punto de vista del niño y del docente. Además, como señalan Adams (1990) y Johnston (1998), a pesar de que existen pocas investigaciones que expliquen el porqué de la eficacia de los métodos de desarrollo de la conciencia fonológica, los estudios que avalan dicha eficacia práctica en el aula son numerosos. Por tanto, concluimos que la intervención didáctica explícita para desarrollar la conciencia fonológica (aunque tal vez no imprescindible) es beneficiosa para el proceso de lectura.

Recogiendo lo dicho hasta ahora, destacamos los aspectos fundamentales para nuestra propuesta respecto a la conciencia fonológica en el aprendizaje de la lectura:

1. Juega un papel crucial en el estadio inicial.

2. Facilita el proceso (aunque según algunos estudios se puede aprender a leer sin conciencia fonológica).

3. Se relaciona con un buen nivel lector.

4. La intervención didáctica explícita para desarrollarla (aunque no es imprescindible) es beneficiosa para el proceso lector.

En el caso de los alumnos de habla inglesa, esta intervención didáctica tiene lugar por medio de los métodos denominados phonics, que se utilizan de manera generalizada en el ámbito anglosajón ${ }^{8}$. Los métodos phonics enseñan el sonido de las palabras y su relación con las grafías, lo que se denominan principios grafo-fonémicos, de modo que el niño capte la estructura ortográfica del inglés. Es decir, se enseña ortografía y fonética al mismo tiempo.

${ }^{8}$ Como hemos dicho antes, la alternativa al uso de phonics la constituyen los métodos globales (en inglés holistico whole language), para su utilización en ámbito anglosajón ver Holdaways (1979). 
La mayoría de estudios se han centrado en el aprendizaje de la lectura en una lengua nativa y no dudamos de que este proceso es diferente en el caso de una lengua extranjera, como es nuestro caso, ya que los alumnos ya conocen el alfabeto y se trataría de ayudarles a que establezcan la relaciones entre las letras y los sonidos de la nueva lengua.

El único trabajo del que tenemos conocimiento con hablantes de inglés como segunda lengua es el trabajo de Stuart (1999). Esta autora realiza un estudio con 96 niños de 5 años que aprenden inglés como segunda lengua en Londres (se trata de niños procedentes de barrios pobres). Utiliza un método comercial para la enseñanza de la lectura (The Phonics Handbook de Lloyd (1992, reeditado en 1995)) y son los propios profesores de los niños quienes les enseñan a leer con este método tras haber recibido previamente instrucciones sobre cómo utilizarlo. Tras la utilización durante 12 semanas del método The Phonics Handbook en el aula, esta autora encuentra efectos positivos claros y significativos en el desarrollo de la lectura y la escritura. Además, demuestra que los profesores solo necesitan una preparación mínima y nada compleja para poder utilizar estos materiales de manera eficaz. Este último aspecto es también de sumo interés para nuestra propuesta, ya que la eficacia de cualquier estrategia de intervención didáctica se debe medir en función de su impacto en el aprendizaje y de sus posibilidades reales de integración en el contexto del aula. En otras palabras, si la utilización de phonics exigiera al docente un esfuerzo que éste no es capaz de asumir nunca se integrarían en el aula de manera sistemática.

Por todo lo dicho anteriormente y a la luz del estudio de Stuart, concluimos que enfrentar a nuestros alumnos a tareas específicas que los fuercen al desarrollo de la conciencia fonológica reportará beneficios al proceso de lecto-escritura en inglés. Del mismo modo, creemos que sus propios profesores son los agentes adecuados para llevar a cabo dicho proyecto.

En el siguiente punto analizamos en profundidad la naturaleza específica de la conciencia fonológica en los alumnos a los que destinamos nuestra propuesta: alumnos españoles que aprenden inglés en Educación Primaria.

\subsection{La enseñanza de la lectura en inglés en Educación Primaria}

El proceso de aprendizaje de la lectura es más complejo en inglés que en castellano. En inglés, la lengua escrita difiere enormemente de la lengua oral. Así, mientras en castellano contamos con 29 letras que representan 24 fonemas; en inglés los alumnos se enfrentan a 26 letras que representan 44 fonemas. Además, contamos con la dificultad añadida de que el inglés presenta enormes irregularidades de pronunciación. De hecho, en el ámbito de habla inglesa, el proceso de enseñanza de la lectura cuenta con infinidad de trabajos científicos y sus consecuentes materiales pedagógicos basados en la conciencia fonológica (Bishop, 1986; Fitzpatrick, 1997; Hohmann, 2002; Jager, Goorman y Llundberg, 1997; Lloyd, 1992, 1995; McCracken y McCracken, 1996 entre otros).

Paradójicamente, en el caso de los alumnos españoles que se enfrentan en Educación Primaria a la lectura de la lengua extranjera, no se produce una enseñanza específica de la misma $^{9}$, sino que únicamente se enseña el alfabeto y la pronunciación de las letras, lo cual

\footnotetext{
${ }^{9}$ Afirmamos esto basándonos en el hecho de que en los materiales didácticos que se utilizan en España para la enseñanza del inglés en Educación Primaria no aparecen métodos ni actividades de enseñanza de la lectura. Por supuesto, es posible que haya maestros que, de manera individual, estén utilizando dichos métodos en el aula
} 
ha quedado demostrado que no es útil para ayudar al proceso lector (Ball y Blachman, 1991). De hecho, el modo de proceder consiste en asumir que al niño que ya ha aprendido a leer en castellano se le puede 'dejar' que transfiera las estrategias lectoras del castellano (regidas por normas bien diferentes) para después ir corrigiendo 'sobre la marcha', de modo que el alumno vaya deduciendo y adquiriendo las normas grafo-fonémicas propias del inglés.

Evidentemente, el resultado es que el proceso lector en inglés es lento, dificultoso y desmotivador para el alumno. Lento, porque el niño cuenta con pocas horas de exposición al idioma y tiene que deducir las complejas normas grafo-fonémicas del inglés que son además diferentes de las del castellano. Dificultoso por esto mismo, porque al proceder por la vía de la transferencia de la competencia lectora en castellano, el niño se ve obligado a un proceso de reajustes de las normas grafo-fonémicas de ambas lenguas que reviste una enorme complejidad. Desmotivador, por lo dicho, por ser un proceso lento y dificultoso. En conclusión, sería sumamente beneficioso facilitar al alumno el paso del texto oral al texto escrito adoptando y adaptando las estrategias de enseñanza lectora utilizadas en el mundo anglosajón que le ayudarían a establecer los patrones de pronunciación del inglés. Como hemos dicho anteriormente, el niño puede, a largo plazo, inferir dichos patrones a base de ser expuesto a textos escritos y orales pero, sin duda, una intervención didáctica explícita que le explique las normas grafo-fonémicas del inglés puede facilitar enormemente el proceso.

A continuación, explicamos con mayor detalle las razones de la complejidad del aprendizaje de la lectura en inglés por parte de nuestros alumnos y cuáles son sus consecuencias negativas para el aprendizaje de dicha lengua.

Los sujetos de nuestro estudio son los alumnos de Primaria que, más o menos (dependiendo del centro), finalizan la etapa de Educación Infantil con el mecanismo de la lectura ya adquirido en castellano, siendo capaces de comprender palabras e incluso frases y textos sencillos en algunos casos. En cuanto al inglés, y también dependiendo del centro, la utilización de textos escritos de manera sistemática se inicia en $2^{\circ}$ o $3^{\text {er }}$ curso de Educación Primaria, después de haber sido expuestos a textos orales en los cursos anteriores. Es decir, cuando se inicia el proceso lector en inglés: los niños son lectores de castellano en un estadio inicial y conocen la pronunciación del inglés, ya que han aprendido esta lengua de manera exclusivamente oral.

Dadas estas características, nuestros alumnos se enfrentan tanto a la complejidad intrínseca de la lectura en inglés como a la complejidad añadida de la transferencia grafo-fonémica del castellano, como mostramos a continuación de manera resumida ${ }^{10}$ :

1. La complejidad intrínseca de la lengua inglesa:

- Un sistema de 26 letras y 44 fonemas.

- Un sistema con numerosas irregularidades.

- Un sistema en el que las relaciones grafía-fonema casi nunca son biunívocas:

(i). Una letra no se corresponde exclusivamente con un fonema:

- la letra 'g' se pronuncia:

\footnotetext{
${ }^{10}$ Presentamos aquí de forma muy resumida y con ejemplos sencillos las dificultades a que se enfrenta un hispanohablante cuando aprende a leer en inglés. Para un estudio contrastivo detallado del inglés y el castellano ver alguno de los manuales de fonología inglesa para hispano hablantes (Alcaraz y Moody, 1999;
} 
- /g / como en 'gate'

- $/ \mathrm{d} 3 /$ como 'gin'

- /3/ como en 'garage'

- /Ø / como en 'sign'

(ii). Un fonema no se corresponde exclusivamente con una letra:

-el fonema $/ \wedge /$ se encuentra representado por las siguientes grafías:

- 'u' como en 'but'

- 'oo' como en 'flood'

- 'o' como en 'love'

- 'ou' como en 'country'

2. La transferencia del castellano:

- Castellano e inglés comparten el alfabeto pero no el inventario de fonemas: en castellano hay 24 y en inglés 44; algunos fonemas son iguales; algunos son parecidos pero diferentes; muchos fonemas del inglés no existen en castellano.

- La relación fonema-letra del castellano no equivale en muchas ocasiones a la relación fonema-letra del inglés, como vemos en el ejemplo de la letra 'a':

(i). Inglés: letra ' $a$ ' sonido:

- $/ \mathfrak{x} /$, como en 'cat'

- /eI/, como en 'cake'

- $/ a: /$, como en 'car'

- /eə/ como en 'care'

- $/$ / como en 'arrive'

- io/ como en 'warrior'

- $/$ :/ como en 'ball'

- /I/ como en 'advantage'

(ii). Castellano: letra ' $\mathbf{a}$ ' sonido:

- / A / como en 'casa'

Por supuesto habría que añadir a estas dificultades de naturaleza fonética el escaso dominio del inglés por parte de nuestros alumnos y el escaso dominio de la lectura en castellano, ya que se encuentran todavía en una etapa inicial del aprendizaje en ambos casos.

Como consecuencia de las diferencias entre ambas lenguas que acabamos de señalar, la transferencia de las estrategias de asociación grafía-sonido del castellano provocará que los alumnos no sean capaces, en etapas iniciales, de establecer la relación entre texto escritotexto oral en inglés. Veamos un ejemplo muy sencillo:

1. El niño conoce y reconoce oralmente una palabra como "ear"/iə/ y la asocia con el objeto correspondiente 8 y con la palabra en castellano "oreja".

2. El niño se enfrenta al texto escrito "ear" y tiene dificultad para vincularlo con el texto oral y con el objeto, ya que al transferir las estrategias de lectura del castellano lee lear/.

Como consecuencia, el niño se ve obligado a utilizar la vía ortográfica o visual para llegar a la comprensión. Esta vía consiste en la asociación directa entre las grafías "ear" y el concepto o realidad a que se refieren $\boldsymbol{9}$. Es decir, el niño memoriza la ortografía de la 
palabra para reconocerla en vez de reconocerla a través de la pronunciación. Como resultado, la fonología queda relegada a un segundo plano en la comprensión lectora en inglés y con ella queda relegada la pronunciación, que se ve perjudicada y, a partir de ahora, "contaminada" por las grafías, ya que el niño, si no se produce una intervención didáctica que lo evite, va a adaptar la pronunciación del inglés a través del texto escrito a las normas ortográficas del castellano.

Por supuesto, somos conscientes de que esta adaptación a la pronunciación de la lengua nativa se produce también cuando la enseñanza se da de modo oral, ya que es una tendencia general en el aprendizaje de una segunda lengua (Gallardo del Puerto, Lecumberri y Cenoz, 2001), pero una cosa es partir de la pronunciación correcta de la palabra y atraerla a los fonemas del castellano y otra cosa bien diferente es que la lectura basada en las relaciones fonema-grafía del castellano propicie directamente una pronunciación incorrecta.

Así pues, sin una intervención didáctica explícita, las consecuencias negativas para el proceso lector en inglés son claras:

1. La complejidad lo convierte en un proceso desmotivador.

2. La pronunciación se ve perjudicada por la transferencia de la asociación grafíasonido del castellano.

3. La comprensión lectora es más costosa en términos de tiempo y esfuerzo.

Por todo ello, resultará beneficiosa la intervención didáctica a través de los phonics que proponemos en este artículo, es decir, ayudar a que los niños establezcan de forma adecuada la relación entre las grafías y los sonidos del inglés, de modo que se beneficie la pronunciación, la comprensión lectora, la propia motivación del alumno y, en última instancia, el nivel de inglés.

A todo lo dicho podemos añadir otras razones que justificarían la viabilidad y el interés de esta propuesta. Por ejemplo, hay que tener en cuenta que el aula es para la mayoría de alumnos el único lugar donde aprenden el idioma, de modo que al contar con tan pocas horas de exposición al inglés es fundamental que la docencia sea lo más eficaz posible. Por otra parte, es en Educación Primaria el mejor momento para el desarrollo de las destrezas de comprensión oral y auditiva, a las que sin duda contribuiría la utilización de phonics, ya que reforzaría la pronunciación del alumno. De una manera más general, también contribuimos a uno de los objetivos fundamentales en esta etapa educativa: el fomento del interés por la lectura en sí misma y por la lengua extranjera, ya que haremos que el proceso lector en inglés sea ameno y asequible tanto para el alumno como para el profesor. A efectos meramente prácticos, la utilización de los phonics es claramente factible, ya que se trata, en realidad, de aprovechar un recurso ya existente, disponible, eficaz y, además, fácil de utilizar por los propios profesores de los alumnos, de modo que la inversión de esfuerzo por parte de los profesores para aprender a enseñar a leer con phonics será mínima en proporción a los logros que esperamos se obtengan con esta intervención didáctica. De hecho, este último aspecto es de suma importancia, ya que es imposible integrar la utilización de una determinada práctica docente si ésta no se adecua a las posibilidades del docente que la ha de utilizar. Finalmente, queremos concluir este apartado enfatizando el hecho de que se trata de una propuesta innovadora, acorde con la situación actual del aula de inglés y con visión de futuro, ya que el inglés va a estar presente no solo en el aula de inglés en Educación Primaria, sino también en otras asignaturas de esta etapa que comienzan a impartirse en inglés y, cada vez más, en Educación 
Infantil, donde los alumnos comienzan a tomar contacto con actividades de pre-lectura, relacionadas con la conciencia fonológica.

Por tanto nuestra propuesta tendría dos objetivos principales y muchos beneficios derivados, como mostramos a continuación:

1. Formar a los Maestros en la utilización de phonics y así:

a. Proporcionarles una nueva herramienta sencilla de utilizar y eficaz.

b. Proporcionarles una nueva herramienta que se adecua al contexto actual del aula de inglés.

2. Facilitar al alumno de Educación Primaria el proceso de aprendizaje de la lectura en inglés a través de la utilización de phonics para:

a. Evitar que el texto escrito interfiera en la pronunciación y proteger y mejorar la pronunciación.

b. Favorecer la lectura comprensiva al asociar sonido-grafía.

Y además:

c. Evitar la desmotivación que se produce en los alumnos ante las dificultades que supone enfrentarse con el texto escrito.

d. Mejorar el nivel de inglés.

e. Aumentar el interés por la nueva lengua a través de la lectura.

f. Aumentar el interés por la lectura en sí misma.

Además de los beneficios centrados en la propia intervención didáctica, nuestra propuesta tendrá también una clara repercusión en otros ámbitos. Por su novedad, será de sumo interés para la Innovación Educativa, tanto desde el punto de vista de la práctica docente como desde el punto de vista puramente científico, donde supondrá una contribución a los numerosos trabajos científicos sobre la enseñanza de la lectura que se realizan desde diferentes áreas de estudio: la Didáctica de las Lenguas Extranjeras, la Adquisición de Lenguas, la Sicología, la Pedagogía y la propia Fonética. Sin duda, servirá también para abrir nuevas vías de investigación que podrán analizar diversos aspectos tales como la posible relación entre la enseñanza lectora en primeras y segundas lenguas, la transferencia de las habilidades lectoras de la lengua nativa, la conexión entre capacidad de análisis fonológico y comprensión lectora en la segunda lengua etc. Además, por su naturaleza, este trabajo supone una importante aportación científica al área de Didáctica de la Lengua Extranjera por el hecho de ofrecer una doble vertiente: científica y práctica, ya que, basándose en los hallazgos de trabajos científicos propone comprobarlos empíricamente aplicándolos al aula de inglés por los propios docentes. Esto es sin duda importante, ya que la práctica docente y el ámbito científico deben ir de la mano y no circular por caminos diferentes como sucede en numerosas ocasiones en las que se desaprovechan los logros de ambos campos.

Si la utilización de los métodos phonics en la enseñanza de la lectura en inglés resulta fructuosa, una clara repercusión sería su inclusión en las programaciones anuales de los centros, así como la adaptación de los propios métodos phonics para aprendices hispanohablantes y la correspondiente elaboración de manuales de enseñanza de inglés que incluyan actividades de concienciación fonológica entre sus contenidos. 
Finalmente, nuestra propuesta no solo responde a la realidad del aula de inglés en España, sino que también responde a las demandas sociales de muchos otros países, donde el aprendizaje de lenguas cobra cada vez mayor importancia y, en concreto, el aprendizaje del inglés como lengua extranjera está presente en todos los niveles educativos. De hecho, la difusión de esta propuesta puede suponer una aportación al Espacio Europeo de Investigación, ya que la enseñanza del inglés está extendida por toda Europa, de modo que los logros se pueden extrapolar a muchos otros contextos europeos.

\section{REFERENCIA BibLiográficas}

Adams, M. J. (1990). Beginning to read. Cambridge, Mass.: MIT Press.

Agencia Nacional de Evaluación de la Calidad y Acreditación (2004). Libro blanco del Título de Grado de Magisterio. Volúmenes I y II. Accesibles a través de: http://www.aneca.es/ modal eval/conver docs titulos.html

Alcaraz, E. y Moody, B. (1999). Fonética inglesa para españoles (4a edición). Alcoy: Marfil.

Alegría, J. y Morais, J. (1979). “'Le developpement de l'habilité d'analyse phonétique de la parole et l'apprentisage de la lecture", en Archives de psychologie, 47: 251-270.

Ball, E. W., y Blachman, B. A. (1991). "Does phoneme awareness training in kindergarten make a difference in early word recognition and developmental spelling?", en Reading Research Quarterly, 24, 1: 49-66.

Bishop, M. M. (1986). The ABC's and All Their Tricks: The Complete Reference Book of Phonics and Spelling. Londres: Mott Media.

Bradley, L. y Bryant, P. E. (1983). "Categorizing sounds and learning to read a causal connection", en Nature, 301: 419-421.

Bryant, P. E. y L. Bradley (1998). Problemas infantiles de lectura. Madrid: Alianza.

Byrne, B. y Fielding-Barnsley, R. (1991). "Evaluation of a program to teach phonemic awareness to young children", en Journal of Educational Psychology, 83: 451-455.

Carrillo, M. S. y Sánchez-Meca, J. (1990). “'Segmentación fonológica-silábica y adquisición de la lectura: un estudio empírico", Comunicación, lenguaje y educación, 9: 109-116.

Cassany, D. (1987). Describir el escribir. Cómo se aprende a escribir. Barcelona: Paidós.

Cassany, D. (1993). Reparar la escritura. Didáctica de la corrección de lo escrito. Barcelona: Graó.

Chall, J. (1983). Learning to Read: The Great Debate. Nueva York: McGraw-Hill.

Clemente, M. (2001). Enseñar a leer. Madrid: Pirámide.

Clemente, M. y Domínguez, A. B. (1999). La enseñanza de la lectura. Enfoque psicololingüístico y sociocultural. Madrid: Pirámide.

Consejo de Europa. Departamento de Política Lingüística (2001). Marco Común Europeo de referencia para las lenguas: aprendizaje, enseñanza, evaluación. Estrasburgo: Consejo de Europa.

Cunningham, A. E. (1990). "Explicit versus implicit Instruction in Phonemic Awareness", en Journal of Experimental Child Psychology, 50: 429-444.

Domínguez, A. B. (1996a). "El desarrollo de habilidades de análisis fonológico a través de programas de enseñanza", en Infancia y Aprendizaje, 76: 69-81.

Domínguez, A. B. (1996b). "Evaluación de los efectos a largo plazo de la enseñanza de habilidades de análisis fonológico sobre el aprendizaje de la lectura y de la escritura", en Infancia y Aprendizaje, 76, 83-96. 
Ehri, L. C. y Wilce, L. S. (1980). "The influence of orthography on readers' conceptualization of the phonemic structure of words", en Applied Psycholinguistics, 1: 371-385.

Ehri, L. C. y Wilce, L. S. (1987). "Does learning to spell help beginners learn to read words?", en Reading Research Quarterly, 18: 47-65.

Finch, D. F. y Ortiz Lira, H. (1982). A course in English phonetics for Spanish speakers. Londres: Heineman.

Fitzpatrick, J. (1997). Phonemic Awareness: Playing With Sounds to Strengthen Beginning Reading Skills. Chipre: Creative Teaching Press.

Gallardo del Puerto, P., García Lecumberri, M. L. y Cenoz, J. (2001). "L3 English vowel and consonant discrimination in learners with different ages of first exposure", en L. Iglesias y S. Doval Suárez (eds.) Studies in Contrastive Linguistics. Proceedings of the $2^{\text {nd }}$ International Contrastive Linguistics Conference. Santiago de Compostela: Universidad de Santiago de Compostela, 419-426.

Graves, D. (1991). Didáctica de la escritura. Madrid: Morata-MEC.

Griffith, P. y Olson, M. W. (1992). "Phonemic Awareness Helps Beginning Readers Break the Code", en Reading Teacher 45, 7: 516-523.

Hohmann, M. (2002). Fee, Fie, Phonemic Awareness: 130 Pre-reading Activities for Preschoolers. Detroit: High/Scope Press.

Holdaway, D. (1979). The foundations of literacy. Sydney, NSW: Ashton Scholastic.

Jager, M. Goorman, B. R. y Llundberg, I. (1997). Phonemic Awareness in Young Children: A Classroom Currículum. Baltimore, M. D.: Brookes Publishing Company.

Jiménez, J. E. y Ortiz, M. R. (1995). Conciencia fonológica y aprendizaje de la lectura. Teoría, evaluación e intervención. Madrid: Síntesis.

Johnston, R. (1998). "Response to Scholes (1998). "The case for orthographic knowledge", en Journal of Research in Reading, 21, 3: 195-200.

Jorm, A. F. y Share, D. L. (1983). "An Invited Article Phonological Recording and Reading Acquisition" en Applied Psycholinguistics, 4, 2: 103-147.

Juel, C., Griffith, P. L. y Gough, P. B. (1986). "Acquisition of literacy: A longitudinal study of children in first and second grade", en Journal of educational psychology, 78: 243-255.

Ley Orgánica de Ordenación General del Sistema Educativo (1/1990, de 3 de Octubre). Publicada en el Boletín Oficial del Estado $\mathrm{n}^{\circ} 238$ de 4 de octubre de 1.990.

Ley Orgánica de Calidad de la Educación (10/2002 de 23 de diciembre). Publicada en el Boletín Oficial del Estado n 307 de 24 de diciembre de 2002.

Ley Orgánica de Educación (2/2006, de 3 de mayo). Publicada en el Boletín Oficial del Estado número 106, de 4 de mayo de 2006.

Liberman, I., Shankweiler, D., Fischer, F. W. y Carter, B. (1974). "Explicit syllable and phoneme segmentation in the young child", en Journal of Experimental Child Psychology, 18: 201212.

Lloyd, S. (1992, 1995). The Phonics Handbook: A Handbook for Teaching Reading, Writing and Spelling. Essex, Reino Unido: Jolly Learning.

Madrid, D y McLaren, N. (2004). TEFL in Primary Education. Granada: Universidad de Granada.

McCracken, M. y McCracken, R. (1996). Spelling Through Phonics. Winnipeg: Peguis Publishers.

Mott, B. (2000). English Phonetics and Phonology for Spanish Speakers. Barcelona: EUB.

Morais, J., Cary, L., Alegría, J. y Bertelson, P. (1979). "Does awareness of speech as a sequence of phones arise spontaneously?", en Cognition, 8: 1-9.

Morais, J., Cluytens, M. y Alegría, J. (1984). "Segmentation abilities of dyslexics and normal readers", en Perceptual and Motor Skills, 58: 221-222. 
Orden Foral 230/1992 de 12 de junio. Publicada en el Boletín Oficial de Navarra ${ }^{\circ} 88$.

Orden Foral 366/2002 de 5 de julio. Publicada en el Boletín Oficial de Navarra ${ }^{\circ} 126$.

Pressley, M. (1999). Cómo enseñar a leer. Barcelona: Paidós.

Read, C., Zhang, Y., Nie, H. y Ding, B. (1986). "The ability to manipulate speech sounds depends on knowing alphabetic reading", en Cognition, 24: 31-44.

Scarborough, H. S.; Ehri, L. C.; Olson, R. K.; y Fowler, A. E. (1998). "The fate of phonemic awareness beyond the elementary school years", en Scientific Studies of Reading, 2: 115142.

Scholes, R. J. (1998). "The case against phonemic awareness", en Journal of Research in Reading 21, 3: 177-188.

Solé, I. (1992). Estrategias de lectura. Barcelona, ICE Universidad de Barcelona: Graó.

Stuart, M. (1999). "Getting ready for reading: Early phoneme awareness and phonics teaching improves reading and spelling in inner-city second language learners", en British Journal of Educational Psychology 69: 587-605.

Teberosky, A. (1991). Aprendiendo a escribir. Barcelona: ICE/Horsori.

Tolchinsky, L. (1993). Aprendizaje del lenguaje escrito. Anthropos: Barcelona.

Tunmer, W. E. (1989). "The role of language-related factors in reading disability", en D. Shankweiler y L. I. Liberman (eds.), Phonology and reading disability: Solving the reading puzzle. Ann Arbor: University of Michigan Press, 91-131.

Vez, J. M., Guillén, C. y Alario, C. (2002). Didáctica de la lengua extranjera en educación infantil y primaria. Madrid: Síntesis. 